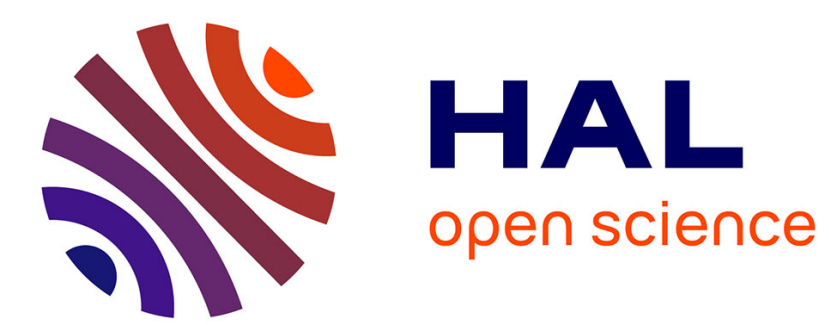

\title{
Geometric modeling of the movement based on an inverse optimal control approach
}

\author{
Frédéric Jean, Paolo Mason, Francesca Chittaro
}

\section{To cite this version:}

Frédéric Jean, Paolo Mason, Francesca Chittaro. Geometric modeling of the movement based on an inverse optimal control approach. 52nd IEEE Conference on Decision and Control CDC 2013, Dec 2013, Florence, Italy. pp.1816-1821, 10.1109/cdc.2013.6760146 . hal-00925297

\section{HAL Id: hal-00925297 \\ https://hal.science/hal-00925297}

Submitted on 7 Jan 2014

HAL is a multi-disciplinary open access archive for the deposit and dissemination of scientific research documents, whether they are published or not. The documents may come from teaching and research institutions in France or abroad, or from public or private research centers.
L'archive ouverte pluridisciplinaire HAL, est destinée au dépôt et à la diffusion de documents scientifiques de niveau recherche, publiés ou non, émanant des établissements d'enseignement et de recherche français ou étrangers, des laboratoires publics ou privés. 


\title{
Geometric modeling of the movement based on an inverse optimal control approach
}

\author{
F. Jean, P. Mason, F.C. Chittaro
}

\begin{abstract}
The present paper analyses a class of optimal control problems on geometric paths of the euclidean space, that is, curves parametrized by arc length. In the first part we deal with existence and robustness issues for such problems and we define the associated inverse optimal control problem. In the second part we discuss the inverse optimal control problem in the special case of planar trajectories and under additional assumptions. More precisely we define a criterion to restrict the study to a convenient class of costs based on the analysis of experimentally recorded trajectories. This method applies in particular to the case of human locomotion trajectories.
\end{abstract}

\section{INTRODUCTION}

This paper is motivated by recent applications of optimal control theory to problem arising from neurophysiology, such as the pointing movements of the arm ([2]), and goal-oriented human locomotion ([5], [6]). Indeed, it is a widely accepted opinion in the neurophysiology community that human movements follow a decision that undergoes an optimality criterion (see [13]). Inverse optimal control is then the appropriate tool for investigating these criteria.

It consists in the following procedure: assume that we are given a set $\Gamma$ of experimental data, and that we define a class of optimal control problems - that is, a pair (control system, class $\mathcal{L}$ of costs) - suitable to model the system. With each cost function in $\mathcal{L}$, one can associate the set of solutions of the corresponding optimal control problem. The inverse optimal control problem consists in determining an inverse of this mapping, in order to determine the cost $L$ such that the minimizing trajectories of the optimal control problem associated with $L$ fit accurately the elements of $\Gamma$.

As we remark in the following, the well-posedness of this problem is not obvious. Nevertheless, for some specific systems, some preliminary results are obtained; we refer to [12], where the issue of the reconstruction of the cost is analyzed, and to [7], which is concerned with continuity properties of the minimizers, with respect to variations of the cost in some particular class.

This work was supported by Digiteo grant Congeo; by the ANR project GCM, program "Blanche", project number NT09_504490; by the Commission of the European Communities under the 7th Framework Programme Marie Curie Initial Training Network (FP7-PEOPLE-2010-ITN), project SADCO, contract number 264735; and by the research fund CARTT, IUT Toulon-La Garde.

F. Jean is with ENSTA ParisTech, Palaiseau, France and team GECO, INRIA Saclay-Île-de-France, France. Frederic. Jeandensta-paristech.fr

P. Mason is with Laboratoire des Signaux et Systèmes, CNRS, Gifsur-Yvette, France and team GECO, INRIA Saclay-Île-de-France, France. paolo.masonelss. supelec. fr.

F. Chittaro is with LSIS - Université du Sud-Toulon Var, France. francesca-carlotta. chittaroeuniv-tln.fr
In this paper, we are concerned with optimal control problems on geometric curves in $\mathbb{R}^{n}$, that is immersed onedimensional submanifold of $\mathbb{R}^{n}$. For every positive integer $k$, $\mathcal{L}_{k}$ denotes the class of costs that depend on the $(k+1)$-th jets of the geometric curves, and that satisfy some coercivity and convexity hypotheses. The class of admissible costs is given by the union of $\mathcal{L}_{k}$, for all positive $k$. With the purpose of investigating the continuity of the minimizers with respect to variations of the cost, we formalize and extend the robustness result of [7] to this more general case, in the following sense: given a suitable family $\left(L_{\varepsilon}\right)_{\varepsilon} \subset \mathcal{L}_{k}$ of costs that converge to some $L_{0} \in \mathcal{L}_{k}$ as $\varepsilon \rightarrow 0$, then the optimal trajectories associated with $L_{\varepsilon}$ converge to the set of minimizers for the cost $L_{0}$ (see Proposition 2.6 and Corollary 2.7).

In [7], we focused on the classes $\mathcal{L}_{1}$ and $\mathcal{L}_{2}$, being them the most appropriate to model goal-oriented human locomotion (see [6]). In particular, we proved a robustness result for sequences of minimizers associated with a sequence of costs belonging to $\mathcal{L}_{2}$ and converging to some $\ell_{0} \in \mathcal{L}_{1}$. In order to further shrink the class of admissible costs, we need to develop a testing procedure, to be applied to experimental data, that points out the differences between syntheses associated with a cost in $\mathcal{L}_{1}$ and syntheses associated with a cost in $\mathcal{L}_{2}$. This test, based on a computation of parametres, is presented in Section III, where also a preliminary analysis on experimental data is briefly presented.

\section{MINIMIZATION PROBLEMS ON GEOMETRIC CURVES}

\section{A. The optimal control problems $\mathbf{P}_{k}(L)$}

We consider the following class of optimization problems. Fix two points $q^{0}, q^{f} \in \mathbb{R}^{n}$ and two unit vectors $v^{0}, v^{f} \in \mathbb{R}^{n}$. The problem is to minimize a cost $J(\gamma)$ on the set of all geometric curves $\gamma$ joining $q^{0}$ to $q^{f}$ and having $v^{0}$ and $v^{f}$ respectively as initial and final directions.

By a geometric curve we mean an immersed onedimensional submanifold of $\mathbb{R}^{n}$. A geometric curve can be parameterized in an intrinsic way by its arc-length, that is $\gamma=q([0, T])$, where $q(\cdot)$ is smooth, $T$ is the length of $\gamma$, and $\dot{q}(t)$ belongs to the unit sphere $S^{n-1} \subset \mathbb{R}^{n}$. To give a geometric curve $\gamma$ is equivalent to give its $(k+1)$-jets $\left(q, \dot{q}, \ldots, q^{(k+1)}\right)(t), t \in[0, T]$, for any nonnegative integer $k$. We will assume that the cost function $J(\gamma)$ is given as the integral of an infinitesimal cost depending on a $(k+1)$-jet of $\gamma$, that is $J(\gamma)=\int_{0}^{T} L\left(q, \ldots, q^{(k+1)}\right) d t$.

Note that the $(k+1)$-jets $\left(q, \dot{q}, \ldots, q^{(k+1)}\right)$ belongs to $\mathbb{R}^{n} \times T^{k} S^{n-1}$, where $T^{k} S^{n-1}$ is the $k$-order tangent bundle of $S^{n-1}$. Since the tangent space $T_{v} S^{n-1}$ may be canonically identified with $(\mathbb{R} v)^{\perp}$ (i.e. the linear subspace of $\mathbb{R}^{n}$ 
orthogonal to $v$ ), the $k$-order tangent bundle of $S^{n-1}$ may be identified with the following subset of $\left(\mathbb{R}^{n}\right)^{k+1}$,

$$
\left\{\left(v_{0}, \ldots, v_{k}\right): v_{0} \in S^{n-1}, v_{i} \in\left(\mathbb{R} v_{0}\right)^{\perp}, i=1, \ldots, k\right\} .
$$

Given a smooth function $u:[0, T] \rightarrow \mathbb{R}^{n}$, any solution $Q(\cdot)=\left(q, v_{0}, \ldots, v_{k-1}\right)(\cdot)$ of the control system

$$
\left\{\begin{aligned}
\dot{q} & =v_{0} \\
\dot{v}_{0} & =v_{1}, \\
& \vdots \\
\dot{v}_{k-1} & =u-\left(v_{0}^{T} u\right) v_{0},
\end{aligned}\right.
$$

with initial values $q(0) \in \mathbb{R}^{n}, v_{0}(0) \in S^{n-1}$, and $v_{1}(0), \ldots, v_{k-1}(0)$ in $\left(\mathbb{R} v_{0}(0)\right)^{\perp}$, is a trajectory on $\mathbb{R}^{n} \times$ $T^{k-1} S^{n-1}$. Hence the control system (1) can be defined on $\mathbb{R}^{n} \times T^{k-1} S^{n-1}$ and any of its trajectory $Q(\cdot)$ is the $k$-jet of the arc-length parameterized curve $q(\cdot)$.

As for the infinitesimal cost $L$, it has to satisfy good properties of positiveness, convexity and coercivity that are summarized in the following definition.

Definition 2.1: Let $k$ be a positive integer and $p>1$ a real number. We define the class $\mathcal{L}_{k}^{p}$ (or $\mathcal{L}_{k}$, for short) as the set of smooth functions $L:\left(\mathbb{R}^{n}\right)^{k+2} \rightarrow \mathbb{R}$ which satisfy the following three assumptions. For every $Q=$ $\left(q, v_{0}, \ldots, v_{k-1}\right) \in \mathbb{R}^{n} \times S^{n-1} \times\left(\mathbb{R}^{n}\right)^{k-1}$ and $v_{k} \in \mathbb{R}^{n}$, there holds:

(H1) $L\left(Q, v_{k}\right) \geq 1$ (i.e., $L$ has a positive lower bound, which may be assumed equal to one up to normalization);

(H2) $\frac{\partial^{2} L}{\partial v_{k}^{2}}\left(Q, v_{k}\right)$ is a positive definite matrix (and so $L$ is strictly convex with respect to the last variable);

(H3) There exist some constants $C, R>0$ such that

$$
\left.L\left(Q, v_{k}\right) \geq C\left|v_{k}\right|^{p} \quad \text { if }\left|v_{k}\right|>R \quad \text { (coercivity of } L\right) .
$$

With these notations, we write our initial minimization problem as an optimal control problem.

$\mathbf{P}_{k}(L)$ Given two pairs $\left(q^{0}, v^{0}\right)$ and $\left(q^{f}, v^{f}\right)$ in $\mathbb{R}^{n} \times$ $S^{n-1}$, minimize

$$
J_{L}=\int_{0}^{T} L(Q(t), u(t)) d t
$$

among all trajectories $Q(\cdot)=\left(q, v_{0}, \ldots, v_{k-1}\right)(\cdot)$ in $\mathbb{R}^{n} \times$ $T^{k-1} S^{n-1}$ of the control system (1) associated with a control $u(\cdot) \in C^{\infty}$ and satisfying $\left(q, v_{0}\right)(0)=\left(q^{0}, v^{0}\right)$ and $\left(q, v_{0}\right)(T)=\left(q^{f}, v^{f}\right)$ (the final time $T$ being free).

\section{B. Analysis of $\mathbf{P}_{k}(L)$}

The first steps in the analysis of an optimal control problem are to prove the existence of optimal solutions and to give necessary conditions of optimality. These steps require a bit of work in our case since our formulation is non standard, the control being chosen in the set of smooth functions.

Theorem 2.2: For every pair $\left(\left(q^{0}, v^{0}\right),\left(q^{f}, v^{f}\right)\right)$ in $\left(\mathbb{R}^{n} \times\right.$ $\left.S^{n-1}\right)^{2}$ with $q^{0} \neq q^{f}$ the problem $\mathbf{P}_{k}(L)$ admits an optimal solution. Moreover, for every optimal solution $Q(\cdot)$ associated with a control $u(\cdot)$, there exists a smooth mapping $P$ : $[0, T] \rightarrow\left(\mathbb{R}^{n}\right)^{k+1}$ which satisfies the following properties.

1. For $t \in[0, T], P(t)=\left(p_{q}(t), p_{0}(t), \ldots, p_{k-1}(t)\right)$ where $p_{q}(t) \in \mathbb{R}^{n}$ and $p_{i}(t) \in\left(\mathbb{R} v_{0}(t)\right)^{\perp}, 0 \leq i \leq k-1$.

$$
\left\{\begin{array}{l}
\dot{p}_{q}=\left(\frac{\partial L}{\partial q}(Q, u)\right)^{T} \\
\dot{p}_{0}=-p_{q}+\left(p_{q}^{T} v_{0}\right) v_{0}+\nabla_{v_{0}}^{t} L(Q, u)(t), \\
\dot{p}_{i}=-p_{i-1}+\nabla_{v_{i}}^{t} L(Q, u)(t), i=1, \ldots, k-1,
\end{array}\right.
$$

where $\nabla_{v_{i}}^{t} L$ denotes the projection on $\left(\mathbb{R} v_{0}(t)\right)^{\perp}$ of the gradient of $L$ with respect to $v_{i}$, i.e.

$$
\nabla_{v_{i}}^{t} L=\left(\frac{\partial L}{\partial v_{i}}\right)^{T}-\left(\frac{\partial L}{\partial v_{i}} v_{0}(t)\right) v_{0}(t)
$$

2. For every $t \in[0, T], p_{k-1}(t)=\nabla_{v_{k}}^{t} L(Q, u)(t)$.

3. For $i=1, \ldots, k-1, p_{i}(0)=p_{i}(T)=0$.

4. The function $H(t)=p_{q}^{T} v_{0}+p_{0}^{T} v_{1}+\cdots+p_{k-1}^{T} u-$ $L(Q, u)$ is identically zero.

The first step of the proof is to establish the existence of an optimal solution when the control $u(\cdot)$ belongs to $L^{p}\left([0, T], \mathbb{R}^{n}\right)$ instead of $C^{\infty}\left([0, T], \mathbb{R}^{n}\right)$, where $p>1$ is the constant arising in the property (H3) satisfied by $L$.

Lemma 2.3: Given two pairs $\left(q^{0}, v^{0}\right)$ and $\left(q^{f}, v^{f}\right)$ in $\mathbb{R}^{n} \times S^{n-1}$ with $q^{0} \neq q^{f}$, there exists a trajectory $Q^{*}(\cdot)$ that minimizes the cost

$$
J_{L}=\int_{0}^{T} L(Q(t), u(t)) d t
$$

among all trajectories $Q(\cdot)$ of the control system (1) with $u(\cdot) \in L^{p}$ satisfying $\left(q, v_{0}\right)(0)=\left(q^{0}, v^{0}\right)$ and $\left(q, v_{0}\right)(T)=$ $\left(q^{f}, v^{f}\right)$.

Proof: The strategy of the proof is very standard. It goes as follows. Consider a minimizing sequence, that is a sequence of trajectories $Q^{N}(\cdot)$ defined on intervals $\left[0, T^{N}\right]$, associated with controls $u^{N}(\cdot) \in L^{p}$, satisfying $\left(q^{N}, v_{0}^{N}\right)(0)=\left(q^{0}, v^{0}\right),\left(q^{N}, v_{0}^{N}\right)\left(T^{N}\right)=\left(q^{f}, v^{f}\right)$, such that $J_{L}\left(Q^{N}, u^{N}\right)$ tends to the infimum value of $\mathbf{P}_{k}(L)$ as $N \rightarrow \infty$. By Hypotheses (H1) and (H3), $T^{N}$ and $\left\|v_{k}^{N}\right\|_{L^{p}}$ are bounded. Therefore, up to subsequences we can assume that $T^{N}$ converges to $\bar{T}$ and that $v_{k}^{N}(\cdot)$ weakly converges to $\bar{v}_{k}(\cdot) \in L^{p}$. Assume that $Q^{N}(\cdot)$ is equicontinuous and uniformly bounded. By Ascoli-Arzelà Theorem, up to subsequences $Q^{N}(\cdot)$ converges uniformly to a trajectory $\bar{Q}(\cdot)$ associated with $\bar{u}(\cdot)=\bar{v}_{k}(\cdot)$. Using the convexity of $L$ (Hypotheses (H2)) and a standard result in calculus of variations [8, Theorem 1.3], we conclude that $\bar{Q}(\cdot)$ is a minimizer of $\mathbf{P}_{k}(L)$.

It is left to prove that $Q^{N}(\cdot)$ is equicontinuous and uniformly bounded. Let us first prove both properties for $v_{k-1}^{N}(\cdot)$. For every $t, t^{\prime} \in\left[0, T^{N}\right]$, we have

$$
\left\|v_{k-1}^{N}(t)-v_{k-1}^{N}\left(t^{\prime}\right)\right\|=\left\|\int_{t}^{t^{\prime}} v_{k}^{N}(s) d s\right\| \leq\left|t-t^{\prime}\right|^{1 / p^{\prime}}\left\|v_{k}^{N}\right\|_{L^{p}},
$$

where $1 / p+1 / p^{\prime}=1$. This implies that $v_{k-1}^{N}(\cdot)$ is $1 / p^{\prime}$ Hölder, and then equicontinuous.

We argue by contradiction and assume that $v_{k-1}^{N}(\cdot)$ is not uniformly bounded. Up to renumbering, we assume that the first coordinate $\left(v_{k-1}^{N}(\cdot)\right)_{1}$ is not uniformly bounded. Using (2) and the fact that $T^{N}$ and $\left\|v_{k}^{N}\right\|_{L^{p}}$ are bounded, we obtain

$$
\left|\left(v_{k-1}^{N}(t)\right)_{1}\right| \geq\left\|\left(v_{k-1}^{N}(\cdot)\right)_{1}\right\|_{L^{\infty}}-\text { const, }
$$


for every $t \in\left[0, T^{N}\right]$. By hypothesis the sequence $a_{N}:=$ $\left\|\left(v_{k-1}^{N}(\cdot)\right)_{1}\right\|_{L^{\infty}}-$ const tends to $+\infty$. Now, by (1), $\left(v_{k-1}^{N}(t)\right)_{1}$ is the derivative of order $k-1$ of $\left(v_{0}^{N}(t)\right)_{1}$. Then (see for instance [6, Lemma 4.10]), there exists a non empty sub-interval of $\left[0, T^{N}\right]$ where $\left|\left(v_{0}^{N}(t)\right)_{1}\right| \geq \operatorname{Const}\left(T^{N}\right)^{k} a_{N}$. Since $v_{0}^{N}(t) \in S^{n-1}$ and $a_{N} \rightarrow \infty$, this implies $\lim T^{N}=0$. But the latter equality contradicts the fact that, for every $N$, $T^{N} \geq\left\|q^{f}-q^{0}\right\| \neq 0$. Thus $v_{k-1}^{N}(\cdot)$ is uniformly bounded.

The same reasoning applies successively to $v_{k-2}^{N}(\cdot)$, $v_{k-3}^{N}(\cdot)$, etc (replacing the $L^{p}$ norm by the $L^{\infty}$ norm in $(2)$ ), and we obtain in this way that $Q^{N}(\cdot)$ is equicontinuous and uniformly bounded, which ends the proof.

Proof of Theorem 2.2: Let $Q^{*}(\cdot)$ be the optimal solution obtained in Lemma 2.3 and denote by $u^{*}(\cdot) \in L^{p}$ the associated control. Without loss of generality we assume $v_{k}^{*}(\cdot)=u^{*}(\cdot)$. Suppose first that we can apply the Pontryagin maximum principle (PMP) stated in a geometrical setting (see [1] for instance) to the solution $Q^{*}(\cdot)$ of the problem $\mathbf{P}_{k}(L)$ in $\mathbb{R}^{n} \times T^{k-1} S^{n-1}$. It is then rather easy to check that there are no abnormal extremals and we thus obtain Conditions $1-5$ with an adjoint mapping $P(\cdot)$ which is absolutely continuous. Moreover, Point 3 ensures that $u^{*}(t)=v_{k}^{*}(t)$ is a smooth function of $p_{k-1}(t)$ and $Q^{*}(t)$ : indeed, Hypothesis (H2) on $L$ implies that $\frac{\partial \nabla_{v_{k}}^{t} L}{\partial v_{k}}$ is invertible and the Implicit Function Theorem applies. As a consequence, Point 2 implies that $\left(Q^{*}, P\right)(\cdot)$ is solution of a smooth ODE and therefore it is a smooth mapping. This gives the whole conclusion of the theorem.

However the classical PMP on a manifold requires that the optimal control $u^{*}(\cdot)$ is bounded in the $L^{\infty}$ topology, which is not assumed here. This difficulty can be overcome in the following way. There exist a finite number of intermediate times $0<T_{1}<\cdots<T_{N}=T$ such that, for every $i=0, \ldots, N-1$, the curve $Q^{*}\left(\left[T_{i}, T_{i+1}\right]\right)$ lies into the domain of some chart of $\mathbb{R}^{n} \times T^{k-1} S^{n-1}$. Each pieces of the trajectory $Q^{*}(\cdot)$ on an interval $\left[T_{i}, T_{i+1}\right]$ should minimize the cost between its extremities. Expressed in coordinates, these pieces satisfy a PMP on an Euclidean space (see for instance [11, Th. 6.2.1]), which only requires that the optimal control is $L^{1}$. Reasoning as previously, the absence of abnormal extremals implies that each pieces of $Q^{*}(\cdot)$ is smooth. In particular the optimal control is bounded and the reasoning above applies.

As noticed in the proof, the problem $\mathbf{P}_{k}(L)$ does not have abnormal extremals. It is rather standard that such a property implies the continuity of the associated value function (see for instance [7, Prop. 2]).

Corollary 2.4: For $\left(\left(q^{0}, v^{0}\right),\left(q^{f}, v^{f}\right)\right)$ in $\left(\mathbb{R}^{n} \times S^{n-1}\right)^{2}$, we define the value function $J_{L}^{*}\left(\left(q^{0}, v^{0}\right),\left(q^{f}, v^{f}\right)\right)$ as the minimum of the problem $\mathbf{P}_{k}(L)$. Then $J_{L}^{*}(\cdot, \cdot)$ is continuous at every pair $\left(\left(q^{0}, v^{0}\right),\left(q^{f}, v^{f}\right)\right)$ such that $q^{0} \neq q^{f}$.

\section{Optimal synthesis and inverse optimal control problem}

We fix now the initial data $\left(q^{0}, v^{0}\right)$ and an annulus $K=$ $\left\{q \in \mathbb{R}^{n}: a \leq\left\|q-q^{0}\right\| \leq b\right\}$ centered at $q^{0}$, where $0<a<b$ are real numbers.
Definition 2.5: The optimal synthesis on $K$ of a cost $L \in \mathcal{L}_{k}$, denoted as $S_{L}$, is defined to be the set of the arc-length parameterized curves $q(\cdot)$ whose $k$-jet $Q(\cdot)=$ $\left(q, \dot{q}, \ldots, q^{(k)}\right)$ is a minimizer of $\mathbf{P}_{k}(L)$ between $\left(q^{0}, v^{0}\right)$ and $(q(T), \dot{q}(T))$ and such that $q(T) \in K$.

Note that, given $L \in \mathcal{L}_{k}$, the length of the curves in $S_{L}$ is uniformly bounded. Indeed, for every $q(\cdot) \in S_{L}$, it follows from hypothesis (H1) that $T \leq J_{L}(Q(\cdot))$, and so that $T \leq J_{L}^{*}\left(\left(q^{0}, v^{0}\right),(q(T), v(T))\right)$. By Corollary 2.4, the function $J_{L}^{*}\left(\left(q^{0}, v^{0}\right), \cdot\right)$ admits a maximum $T_{L}^{*}$ on the compact set $K \times S^{n-1}$ and we thus obtain the uniform bound $T \leq T_{L}^{*}$ for the length $T$ of a curve in $S_{L}$.

We extend every curve $q(\cdot) \in S_{L}$ to the whole interval $[0,+\infty)$ setting $q(t) \equiv q(T)$ for $t \geq T$. Thus $S_{L}$ appears as a subset of $C^{0}\left([0,+\infty), \mathbb{R}^{n}\right)$ that we endow with a distance $d_{\infty}$ associated with the uniform convergence on compact subsets of $[0,+\infty)$ (see e.g. [10, Th. 1.3.2]). Then the set $\mathcal{S}_{k}$ of all optimal synthesis $S_{L}, L \in \mathcal{L}_{k}$, may be endowed with the Hausdorff distance $\operatorname{dist}_{H}$ induced by $d_{\infty}$, that is:

$\operatorname{dist}_{H}\left(S_{L}, S_{L^{\prime}}\right)=\max \left\{\sup _{\gamma \in S_{L}} \inf _{\gamma^{\prime} \in S_{L^{\prime}}} d_{\infty}\left(\gamma, \gamma^{\prime}\right), \sup _{\gamma^{\prime} \in S_{L^{\prime}}} \inf _{\gamma \in S_{L}} d_{\infty}\left(\gamma, \gamma^{\prime}\right)\right\}$.

On the other hand, $\mathcal{L}_{k}$ is a subset of the set of continuous functions on $\left(\mathbb{R}^{n}\right)^{k+1}$, and it must be endowed with a topology similar to the one of the convergence on compact subsets, but which moreover preserves the coercivity property of the costs. Let $d_{c c}$ be a distance associated with the convergence on compact subsets of $\left(\mathbb{R}^{n}\right)^{k}$, then we define the distance $d_{c}$ on $\mathcal{L}_{k}$ by:

$$
d_{c}\left(L, L^{\prime}\right)=\sup _{u \in \mathbb{R}^{n}} d_{c c}\left(\frac{L(\cdot, u)}{1+|u|^{p}}, \frac{L^{\prime}(\cdot, u)}{1+|u|^{p}}\right) .
$$

We finally introduce the map $\Phi: L \mapsto S_{L}$, which associates with a cost $L \in \mathcal{L}_{k}$ its synthesis $S_{L} \in \mathcal{S}_{k}$. The construction of an inverse mapping $\Phi^{-1}$, that is the determination of a cost function from an optimal synthesis, is called an inverse optimal control problem. For such an inverse problem, the main questions concern the wellposedness of the problem:

- is $\Phi$ injective? (note that, by definition, $\mathcal{S}_{k}=\Phi\left(\mathcal{L}_{k}\right)$, and so injectivity is equivalent to invertibility);

- if $\Phi$ is injective, is $\Phi^{-1}$ continuous as a mapping from the metric space $\left(\mathcal{S}_{k}, \operatorname{dist}_{H}\right)$ to the metric space $\left(\mathcal{L}_{k}, d_{c}\right) ?$

We are at the moment far from being able to answer these difficult questions. However we will study two problems that constitutes a first step in that direction:

- given a synthesis $S$, is it possible to recover the value $k \in \mathbb{N}$ such that $S \in \mathcal{S}_{k}$ ?

- is $\Phi$ continuous as a function from the metric space $\left(\mathcal{L}_{k}, d_{c}\right)$ to the metric space $\left(\mathcal{S}_{k}, \operatorname{dist}_{H}\right)$ ?

The first problem, which is a preliminary to the question of injectivity of $\Phi$, will be addressed in the next section for a special case. The second problem concerns the robustness of the direct problem $\mathbf{P}_{k}(L)$ with respect to perturbations of the cost. We have the following partial answer to this question.

Given $\left(q^{f}, v^{f}\right)$ in $\mathbb{R}^{n} \times S^{n-1}$ and a cost $L \in \mathcal{L}_{k}$, we use $\mathcal{M}\left(L ; q^{f}, v^{f}\right)$ to denote the set of trajectories $Q(\cdot)$ minimiz- 
ing the problem $\mathbf{P}_{k}(L)$ between $\left(q^{0}, v^{0}\right)$ and $\left(q^{f}, v^{f}\right)$ (recall that the initial data $\left(q^{0}, v^{0}\right)$ have been fixed).

Proposition 2.6: Fix $\left(q^{f}, v^{f}\right)$ in $\mathbb{R}^{n} \times S^{n-1}$ with $q^{0} \neq q^{f}$. Let $L_{\varepsilon}, \varepsilon \geq 0$, be a family of costs in $\mathcal{L}_{k}$ converging to a cost $L_{0} \in \mathcal{L}_{k}$ as $\varepsilon \rightarrow 0$, and $\left(q^{\varepsilon}, v^{\varepsilon}\right), \varepsilon \geq 0$, be a family of elements of $\mathbb{R}^{n} \times S^{n-1}$ converging to $\left(q^{f}, v^{f}\right)$. Then, for any family of trajectories $Q^{\varepsilon}(\cdot) \in \mathcal{M}\left(L_{\varepsilon} ; q^{\varepsilon}, v^{\varepsilon}\right)$, we have

$$
\lim _{\varepsilon \rightarrow 0} d_{\infty}\left(Q^{\varepsilon}(\cdot), \mathcal{M}\left(L_{0} ; q^{f}, v^{f}\right)\right)=0 .
$$

This result is a generalization of [7, Th. 5]. Its proof will appear in a forthcoming paper.

Corollary 2.7: Let $L_{\varepsilon}, \varepsilon \geq 0$, be a family of costs in $\mathcal{L}_{k}$ converging to a cost $L_{0} \in \mathcal{L}_{k}$ as $\varepsilon \rightarrow 0$. Then

$$
\lim _{\varepsilon \rightarrow 0} \sup _{\gamma^{\varepsilon} \in S_{L_{\varepsilon}}} \inf _{\gamma \in S_{L_{0}}} d_{\infty}\left(\gamma, \gamma^{\varepsilon}\right)=0 .
$$

This result is a first step toward the proof that $\operatorname{dist}_{H}\left(S_{L_{\varepsilon}}, S_{L_{0}}\right) \rightarrow 0$, and so that $\Phi$ is continuous.

\section{Classes of OPTIMAL CONTROL PROBlems FOR THE ORIENTED LOCOMOTION}

In recent years the problem of modeling the oriented human locomotion as the result of an optimization procedure has received an increasing attention (cf. [3], [4], [5], [6], [7], [9]). Different approaches have been attempted. Typically one assumes a simplified kinematic model of movement, which does not take into account all possible degrees of freedom (for instance the dynamics of muscles). In robotics, the complete generation of movement is then obtained through a pseudo-inverse approach starting from the motion planning at the kinematic level.

The simplest model to describe geometric curves on the plane is given by the following equation

$$
\left\{\begin{array}{l}
\dot{x}=\cos \theta \\
\dot{y}=\sin \theta \\
\dot{\theta}=u
\end{array},\right.
$$

where $(x, y)$ denotes the position on the plane and $\theta$ the direction. Alternatively, this equation describes planar trajectories with constant forward velocity equal to one. As observed in [3], for human locomotion the angle $\theta$ can realistically describe the orientation of the body, at least when locomotion trajectories connect far enough initial and final points. That is to say, human locomotion can be described rather precisely via a non-holonomic model.

Notice that Equation (3) is a special case of Equation (1) with $n=2, k=1$. The idea is then to assume that human locomotion trajectories can be identified with solutions of (3) minimizing a certain (integral) cost. A natural cost can for instance correspond to a compromise between time minimization and minimization of an energy term. Inspired by this idea, in [6], [7] we introduced the general class of costs of the following form

$$
J_{L}=\int_{0}^{T} L\left(\dot{\theta}, \ldots, \theta^{(k)}\right) d t
$$

to be minimized among all admissible regular enough $u(\cdot)$ steering system (3) from $q(0)=\left(x_{0}, y_{0}, \theta_{0}\right)$ to $q(T)=$ $\left(x_{1}, y_{1}, \theta_{1}\right)$, where we assume that the hypotheses (H1)-(H3) are satisfied. Note that the cost associated with a trajectory of the system is invariant by roto-translations.

In order to express the optimal control problem in a convenient way, we rewrite Equation (3) as

$$
\left\{\begin{array}{l}
\dot{x}=\cos \theta \\
\dot{y}=\sin \theta \\
\theta^{(k)}=u
\end{array},\right.
$$

so that the optimal control problem fall within the setting of Section II, with $n=2$. Note that planar solutions of (4) coincide with regular enough solutions of (3).

As in Section II we use $\mathbf{P}_{k}(L)$ to denote the class of optimal control defined above and we let $\mathcal{L}_{k}$ be the set of admissible cost functions. The corresponding set of optimal syntheses is denoted as $\mathcal{S}_{k}$.

The problem we are interested in in this section is the following.

Given a synthesis (or a family of optimal trajectories) for an optimal control problem of the previous kind, is it possible to determine the corresponding class of costs $\mathcal{L}_{k}$ ?

We next provide a partial answer to this question by introducing a criterion to distinguish between optimal syntheses for costs in the classes $\mathcal{L}_{1}$ and $\mathcal{L}_{2}$.

\section{A. The optimal front from an inflection point and a discrim- inating criterion}

Let $S$ be an optimal synthesis and consider the subset of $S$ made by all the trajectories such that $\dot{\theta}$ vanishes at least once. We can assume either that $\theta(\cdot) \equiv 0$ or that, up to a roto-translation and a time translation, $t=0$ is the first time such that $\dot{\theta}$ vanishes, and $x(0)=y(0)=\theta(0)=0$ for all the considered trajectories. For a fixed final time $T$ we consider the set $\mathcal{F}_{T}$ of all points $(x, y, \theta)$ that can be obtained evaluating trajectories of $S$ at time $T$ (whenever $T$ is in the corresponding time domain).

Proposition 3.1: Assume that $L(0)=1$ (i.e. 0 is the minimum of $L$ ) and let $S \in \mathcal{S}_{1}$. Then $\mathcal{F}_{T}$ is contained in a one-parametric subset of $\mathbb{R}^{2} \times S^{1}$.

Proof: We apply the Pontryagin maximum principle. The corresponding Hamiltonian function, identically zero along optimal trajectories, is $H=p_{x} \cos (\theta)+p_{y} \sin (\theta)+$ $p_{\theta} u-L(u)$. The adjoint equation associated with the optimal control problem reads

$$
\left\{\begin{array}{l}
\dot{p}_{x}=\dot{p}_{y}=0 \\
\dot{p}_{\theta}=p_{x} \sin (\theta)-p_{y} \cos (\theta)
\end{array},\right.
$$

and the optimal control satisfies the equation

$$
\frac{d L}{d u}(u)=p_{\theta}
$$

Thus it must be $p_{\theta}(0) \stackrel{d u}{=} u(0)=\theta(0)=0$, and evaluating the Hamiltonian at time $t=0$ we get $p_{x}(0)-1=0$. We deduce that all points in $\mathcal{F}_{T}$ are the projection on $\mathbb{R}^{2} \times S^{1}$ of the solution at time $T$ of the system (3)-(5) (with $u$ obtained by inverting (6)) starting from some initial datum $\left(0,0,0,1, p_{y}(0), 0\right)^{T}$. Being the flow a local diffeomorphism, its evaluation at time $T$ is a smooth one-dimensional immersed submanifold of $T^{*}\left(\mathbb{R}^{2} \times S^{1}\right)$ parameterized by $p_{y}(0)$. The proposition is a straightforward consequence. 
We will consider in the next section the case $k=2$. Our purpose is to show that it is always possible to determine if a synthesis $S \in \mathcal{S}_{1} \cup \mathcal{S}_{2}$ belongs to $\mathcal{S}_{1}$ or to $\mathcal{S}_{2}$, and to prove this fact we will show that for any $S \in \mathcal{S}_{2}$ the corresponding set $\mathcal{F}_{T}$ contains a two dimensional submanifold of $\mathbb{R}^{2} \times S^{1}$. By virtue of Proposition 3.1 we thus establish a relevant qualitative difference between syntheses in $\mathcal{S}_{1}$ and $\mathcal{S}_{2}$.

Let us first show, via the following rather general result, that the set of trajectories such that $\dot{\theta}$ vanishes at some time is non empty.

Lemma 3.2: Let $L \in \mathcal{L}_{k}$ for some $k$ and assume moreover that $L(0)=1$. Then there exists an open subset $\Omega$ of $\mathbb{R}^{2} \times$ $S^{1}$ such that every optimal trajectory of $\mathbf{P}_{k}(L)$ joining 0 to a point of $\Omega$ is such that $\dot{\theta}(t)=0$ for some $t$ in the corresponding time domain. Moreover the boundary of $\Omega$ contains the half line $\{(x, 0,0): x>0\}$.

Proof: Fix $x>0$. A trivial consequence of Proposition 2.6 is that, for small $\varepsilon>0$, optimal trajectories reaching the point $(x, 0, \varepsilon)$ are uniformly close, on their time domain $\left[0, T_{\varepsilon}\right]$, to the trajectory $t \mapsto(t, 0,0)$. Since $\theta\left(T_{\varepsilon}\right)>0$, $\int_{0}^{T_{\varepsilon}} \sin \theta(\tau) d \tau=0$ and $\theta(\cdot)$ can be made arbitrarily close to 0 by choosing a suitably small $\varepsilon$ we deduce that $\theta(\cdot)$ must be negative for some $\hat{t} \in\left[0, T_{\varepsilon}\right]$. Thus $\dot{\theta}(t)=0$ for some $t \in\left[\hat{t}, T_{\varepsilon}\right]$. A simple application of Proposition 2.6 shows that the same property must hold for final points in an open neighborhood $\Omega_{x, \varepsilon}$ of $(x, 0, \varepsilon)$. The proof is completed taking $\Omega=\cup_{x>0, \varepsilon} \Omega_{x, \varepsilon}$, for suitably small values of $\varepsilon$.

\section{B. A qualitative property of optimal syntheses of $\mathcal{S}_{2}$}

In this section we state and sketch the proof of a result showing a property of syntheses of $\mathcal{S}_{2}$ which never holds for syntheses in $\mathcal{S}_{1}$, providing an important qualitative difference between the families $\mathcal{S}_{1}$ and $\mathcal{S}_{2}$. Before stating the result we write down the Pontryagin maximum principle associated with the problem. We first rewrite Eq. (3) as follows:

$$
\left\{\begin{array}{l}
\dot{x}=\cos \theta \\
\dot{y}=\sin \theta \\
\dot{\theta}=\kappa \\
\dot{\kappa}=u
\end{array} .\right.
$$

The Hamiltonian function is $H=p_{x} \cos (\theta)+p_{y} \sin (\theta)+$ $p_{\theta} \kappa+p_{\kappa} u-L(\kappa, u)$, and $H \equiv 0$ along optimal trajectories. The adjoint equation is the following

$$
\left\{\begin{array}{l}
\dot{p}_{x}=\dot{p}_{y}=0 \\
\dot{p}_{\theta}=p_{x} \sin (\theta)-p_{y} \cos (\theta) \\
\dot{p}_{\kappa}=-p_{\theta}+\frac{\partial L}{\partial \kappa}(\kappa, u)
\end{array},\right.
$$

and the optimal control satisfies the equation

$$
\frac{\partial L}{\partial u}(\kappa, u)=p_{\kappa}
$$

Consider a trajectory of $\mathbf{P}_{2}(L)$ starting from the point $0 \in \mathbb{R}^{2} \times S^{1}$. It can be obtained by projecting onto the state space $\mathbb{R}^{2} \times S^{1} \times \mathbb{R}$ a solution of (7)-(8) where $u$ is the smooth function of $\kappa$ and $p_{\kappa}$ obtained by inversion of Eq. (9), that we denote as $u\left(\kappa, p_{\kappa}\right)$. Being $\kappa(0)$ free, we obtain the transversality condition $p_{\kappa}(0)=0$. Evaluating the Hamiltonian at the initial point we also get

$$
p_{x}=-p_{\theta}(0) \kappa(0)+L(\kappa(0), u(\kappa(0), 0)) .
$$

Thus, the initial value for (7)-(8) is a smooth function of the parameters $\kappa(0), p_{y}, p_{\theta}(0)$. In particular, for $\mathbf{r}=$ $\left(r_{1}, r_{2}, r_{3}\right) \in \mathbb{R}^{3}$ we denote by $\Phi(\mathbf{r})(\cdot)$ the solution of (7)-(8) with $\left(\kappa(0), p_{y}, p_{\theta}(0)\right)=\mathbf{r}$ and we define a trajectory $\hat{\Phi}(\mathbf{r})(\cdot)$ as the corresponding projection on $\mathbb{R}^{2} \times S^{1}$.

Any solution of $\mathbf{P}_{2}(L)$ must additionally satisfy the transversality condition $p_{\kappa}(T)=0$ at the final time $T$.

We will need the following assumptions.

(H4) The Hessian of $L$ with respect to $(\kappa, u)$ is positive definite at $(0,0)$ and $L(0,0)=1$ (going straight is less expensive than any other kind of motion).

(H5) There exist two open sets, $Q \subset \mathbb{R}^{3}$ and $\hat{\Omega} \subset \mathbb{R}^{2} \times S^{1}$ with $(x, 0,0) \in \hat{\Omega}$ for some $x>0$, such that for any point $z \in \hat{\Omega}$ there exists a unique value $\mathbf{r} \in Q$ such that $\hat{\Phi}(\mathbf{r})(\cdot)$ reaches $z$ and the corresponding value $p_{\kappa}$ vanishes.

Note that under the hypothesis (H4) the straight line is optimal and corresponds to $\hat{\Phi}(0)(\cdot)$. Condition (H5), although very difficult to check, appears to be very natural (for instance it is true for any final point $(x, 0,0) \in \mathbb{R}^{2} \times S^{1}$ ).

We are now ready to state the main result of this section.

Proposition 3.3: Assume (H1)-(H5) hold, and let $S \in \mathcal{S}_{2}$. Then there exists a set $\mathcal{T} \subset[0,+\infty)$ of isolated points such that, for every $T>0$ small enough, $T \notin \mathcal{T}, \mathcal{F}_{T}$ contains a 2-dimensional submanifold of $\mathbb{R}^{2} \times S^{1}$.

In the following we are interested only on solutions of $\mathbf{P}_{2}(L)$ which are different from $\hat{\Phi}(0)(\cdot)$, and which contain a point where $\dot{\theta}$ vanishes. We denote by $Q \subset \mathbb{R}^{3}$ the set of parameters such that the trajectories $\hat{\Phi}(\mathbf{r})(\cdot)$ have this property for $\mathbf{r} \in Q$. Notice that, by assumption (H5), we can identify optimal trajectories with solutions of the Pontryagin maximum principle corresponding to parameters $\mathbf{r}$ in $Q$.

The main idea of the proof is the following: first of all, we prove that the first time where $\dot{\theta}=0$ depends smoothly on $\mathbf{r} \in Q$; then, that the map that sends $\mathbf{r}$ to the values of $\left(p_{y}, p_{\theta}, p_{\kappa}\right)$ corresponding to this time is a diffeomorphism; finally, that $\mathcal{F}_{T}$ contains the range of a smooth map $\Psi_{T}$ of $\left(p_{y}, p_{\theta}, p_{\kappa}\right)$, which has at least rank 2. All these claims are true up to suitable restriction of the domains.

Here below we give more details on the proofs. The complete proof will appear in a forthcoming paper.

As anticipated above, we prove the existence of a two dimensional submanifold of $\mathcal{F}_{T}$, close to a point of the straight line $\hat{\Phi}(0)(\cdot)$. In particular, we consider solutions of $\mathbf{P}_{2}(L)$ starting from the point $0 \in \mathbb{R}^{2} \times S^{1}$ and reaching points arbitrarily close to $\hat{\Phi}(0)(T)$, for some $T>0$. Using the continuous dependence of solutions of (7)-(8) with respect to the initial datum, one can easily get that such trajectories correspond to values $\mathbf{r} \in Q$ belonging to a small neighborhood of 0 (see also [7, Th. 4]). Thus, to study behaviour of the trajectories for small times, we consider the linearization of (7)-(8) around the trajectory $\hat{\Phi}(0)(\cdot)$.

The following result is obtained applying recursively the implicit function theorem. More details are in the appendix.

Lemma 3.4: There exist an open set $\hat{\Omega} \subset \Omega$, an open set $\hat{Q} \subset Q$ contained in a small neighborhood of 0 , and a smooth function $\tau: Q \rightarrow \mathbb{R}$ such that every solution of $\mathbf{P}_{2}(L)$, 


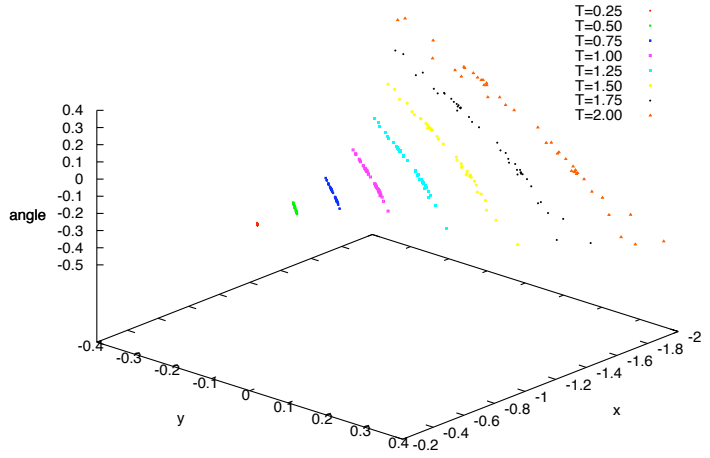

Fig. 1. $\mathcal{F}_{T}$ for different values of $T$.

starting from the origin and reaching a point in $\hat{\Omega}$ at some time $\hat{t}$, coincides with $\hat{\Phi}(\mathbf{r})(\cdot)$ for some $\mathbf{r} \in \hat{Q}$, and $\hat{t}=\tau(\mathbf{r})$. For $\mathbf{r} \in Q$, let $\tau_{0}(\mathbf{r})$ be the smallest positive time such that $\dot{\theta}$ vanishes. Then we have the following result.

Lemma 3.5: Up to restricting once again the set of parameters $\hat{Q}$, the function $\tau_{0}(\cdot)$ is smooth.

In order to analyze the set $\mathcal{F}_{T}$, we perform a roto-translation of the trajectories sending $\hat{\Phi}(\mathbf{r})(\tau(\mathbf{r}))$ into the origin, for any $\mathbf{r} \in \hat{Q}$. This coordinate change induces a corresponding transformation on the cotangent space, namely the new value for the component $p_{y}$ becomes $\bar{p}_{y}(\mathbf{r})=$ $-p_{x}(\mathbf{r}) \sin \left(\theta\left(\tau_{0}(\mathbf{r})\right)\right)+r_{2} \cos \left(\theta\left(\tau_{0}(\mathbf{r})\right)\right)$, where $p_{x}(\mathbf{r})$ is given by (10) and $\theta(\cdot)$ is the angular component of $\hat{\Phi}(\mathbf{r})(\cdot)$. Then, since $\kappa(\tau(\mathbf{r}))=0$ and since the Hamiltonian is identically zero, the new initial condition for (7)-(8) is completely determined by the values $\bar{p}_{y}(\mathbf{r}), p_{\theta}(\tau(\mathbf{r})), p_{\kappa}(\tau(\mathbf{r}))$.

Lemma 3.6: Up to a further restriction of $\hat{Q}$, the map $\varphi(\mathbf{r})=\left(\bar{p}_{y}(\mathbf{r}), p_{\theta}(\tau(\mathbf{r})), p_{\kappa}(\tau(\mathbf{r}))\right)$ is a local diffeomorphism from $\hat{Q}$ to $U=\varphi(\hat{Q})$.

Let $\mathbf{p} \in U$, and consider the solution of (7)-(8) emanating from the origin, with $\kappa=0$ and initial values of the adjoint vectors equal to $\left(p_{y}, p_{\theta}, p_{\kappa}\right)=\mathbf{p}$. Fix some small enough time $t$. We denote with $\Psi_{t}(\mathbf{p})$ the projection on the state space $\mathbb{R}^{2} \times S^{1}$ of the solution corresponding to $\mathbf{p}$, evaluated at the time $t$. Then it is possible to prove the following result.

Lemma 3.7: The differential of $\Psi_{t}$ has rank at least two at any point of $U$.

Proposition 3.3 readily follows from Lemma 3.7 , being the range of $\Psi_{t}$ a subset of $\mathcal{F}_{t}$.

Figure 1 has been obtained processing the experimental data recorded by Arechavaleta et al. (see e.g. [4]) on human locomotion trajectories. It depicts the corresponding values of $(x(t), y(t), \theta(t))$, at several times $t$, after the application of the roto-translation that sends the point corresponding to $\kappa=0$ to $(0,0,0,0)$, and its corresponding time to 0 . Even if, at a first glance, these data seem to suggest that the cost has to be seeked in the class $\mathcal{L}_{1}$, a finer analysis is needed. Indeed, in order to correctly apply the criterion, a numerical estimate of the size of the front $\mathcal{F}_{T}$ for costs in the class $\mathcal{L}_{2}$, which is at the present time unavailable, seems to be necessary.

\section{APPENDIX}

Linearization around the straight line.

In this section we use the following notations

$L_{u u}=\frac{\partial^{2} L}{\partial u^{2}}(0,0), L_{u \kappa}=\frac{\partial^{2} L}{\partial u \partial \kappa}(0,0), L_{\kappa \kappa}=\frac{\partial^{2} L}{\partial \kappa^{2}}(0,0)$.

By taking into account only the dynamics of the variations on the variables $\theta, \kappa, p_{\theta}, p_{\kappa}$ (the others being trivial or non relevant for our analysis) we ends up with the system

$$
\left(\begin{array}{c}
\dot{\delta_{\theta}} \\
\dot{\delta_{\kappa}} \\
\dot{\delta}_{p_{\theta}} \\
\dot{r}_{p_{\kappa}}
\end{array}\right)=\left(\begin{array}{cccc}
0 & 1 & 0 & 0 \\
0 & -\frac{L_{\kappa u}}{L_{u u}} & 0 & \frac{1}{L_{u u}} \\
1 & 0 & 0 & 0 \\
0 & L_{\kappa \kappa}-\frac{L_{\kappa u}^{2}}{L_{u u}} & -1 & \frac{L_{\kappa u}}{L_{u u}}
\end{array}\right)\left(\begin{array}{c}
\delta_{\theta} \\
\delta_{\kappa} \\
\delta_{p_{\theta}} \\
\delta_{p_{\kappa}}
\end{array}\right)-\delta_{p_{y}}\left(\begin{array}{l}
0 \\
0 \\
1 \\
0
\end{array}\right)
$$

We call $\Delta(t)=\left(\delta_{\theta}, \delta_{\kappa}, \delta p_{\theta}, \delta p_{\kappa}\right)^{T}$ and

$$
B=\left(\begin{array}{cccc}
0 & 1 & 0 & 0 \\
0 & -\frac{L_{\kappa u}}{L_{u u}} & 0 & \frac{1}{L_{u u}} \\
1 & 0 & 0 & 0 \\
0 & L_{\kappa \kappa}-\frac{L_{\kappa u}^{2}}{L_{u u}} & -1 & \frac{L_{\kappa u}}{L_{u u}}
\end{array}\right)
$$

The matrix $B$ is invertible being its determinant equal to $1 / L_{u u}$, and we have

$$
\Delta(t)=e^{t B} \Delta(0)-\delta p_{y}\left(I-e^{t B}\right) B^{-1} e_{3}
$$

where $\boldsymbol{e}_{3}$ belongs to the canonical basis of $\mathbb{R}^{4}$. For fixed time $t$, the variation $\Delta(t)$ is thus a linear function of the variations $\Delta(0)$ and $\delta p_{y}$ on the initial condition.

The result in Section III-B are obtained by applying systematically the implicit function theorem to several maps defined on the sets of parameters $\hat{Q}, U$ and on the time domain. The use of this tool is permitted by the surjectivity, up to isolated time values, of suitable projections of the previous linear map (possibly restricted to suitable subspaces), which in particular turns out to be a consequence of the assumption (H4).

\section{REFERENCES}

[1] A.A. Agrachev and Yu.L. Sachkov. Control theory from the geometric viewpoint, Springer-Verlag, Berlin, 2004.

[2] J.-P. Gauthier, B. Berret and F. Jean A biomechanical inactivation principle, Proceedings of the Steklov Institute of Mathematics, 2008.

[3] G Arechavaleta, J.-P. Laumond, H. Hicheur, and A. Berthoz. On the nonholonomic nature of human locomotion. Autonomous Robots, 25:25-35, 2008.

[4] G Arechavaleta, J.-P. Laumond, H. Hicheur, and A. Berthoz. An optimality principle governing human walking. IEEE Transactions on Robotics, 24(1):5-14, 2008.

[5] T. Bayen, Y Chitour, F. Jean, and P. Mason. Asymptotic analysis of an optimal control problem connected to the human locomotion. In Joint 48th IEEE Conference on Decision and Control and 28th Chinese Control Conference, Shanghai, 2009.

[6] Y. Chitour, F. Jean, and P. Mason. Optimal control models of the goal-oriented human locomotion. SIAM Journal on Control and Optimization, 50:147-170, 2012.

[7] F.C. Chittaro, F. Jean, and P. Mason. On the inverse optimal control problems of the human locomotion: stability and robustness of the minimizers. Journal of Mathematical Sciences, to appear.

[8] B. Dacorogna. Direct methods in the calculus of variations. SpringerVerlag New York, Inc., New York, NY, USA, 1989.

[9] K. Mombaur, A. Truong, and J.-P. Laumond. From human to humanoid locomotion - an inverse optimal control approach. Autonomous Robots, 28:369-383, 2010.

[10] J. L. Schiff, Normal families, Springer-Verlag, 1993.

[11] R. B. Vinter, Optimal Control Systems and Control: Foundations and Applications, Modern Birkhäuser classics, Springer, 2000.

[12] A. Ajami, J.-P. Gauthier, T. Maillot and U. Serres, How humans fly, ESAIM COCV (2013)

[13] E. Todorov. Optimal control theory, chapter 12, pages 269-298. Bayesian Brain: Probabilistic Approaches to Neural Coding, Doya K (ed), 2006. 